\title{
A THEOREM OF STOUT
}

\author{
BY MYRON GOLDSTEIN ${ }^{1}$ AND T. R. CHOW $^{2}$
}

Communicated by Frederick Gehring, April 21, 1969

In a paper that appeared in Mathematische Annalen in 1968, E. L. Stout proved a generalization of a theorem of Rado which states that if $f$ is continuous on the set $D=\{z:|z|<1\}$ and holomorphic on $D-f^{-1}(0)$, then $f$ is holomorphic on $D$. Stout's generalization is as follows:

TheOREM. Let $E$ be a set of capacity zero in the complex plane, and let $E_{0}$ be a relatively closed set in $D$. If $f$ is a function bounded and holomorphic in $D-E_{0}$, if $f^{\prime}$ does not vanish identically, and if for every sequence $\left\{z_{n}\right\}$ in $D-E_{0}$ such that $z_{n} \rightarrow z_{0} \in E_{0}$ and $\lim f\left(z_{n}\right)=w_{0}$ exists it is the case that $w_{0} \in E$, then $f$ is holomorphic throughout $D$.

We shall generalize this theorem by replacing $D$ by an arbitrary hyperbolic Riemann surface and replacing the assumption that $f$ is bounded by the assumption that $f$ belong to the Hardy class $H^{P}$, $0<P \leqq \infty$, i.e., that $|f|^{P}$ possesses a harmonic majorant. Thus our theorem reads as follows:

THEOREM. Let $E$ be a set of capacity zero in the complex plane, and let $E_{0}$ be a closed subset of a hyperbolic Riemann surface $R$. If $f \in H^{P}\left(R-E_{0}\right)$, i.e., $f$ is of class $H^{P}$ on each component of $R-E_{0}$, $0<P \leqq \infty$, if $f$ is nonconstant on some component of $R-E_{0}$, and if for every sequence $\left\{a_{n}\right\}$ in $R-E_{0}$ such that $a_{n} \rightarrow a_{0} \in E_{0}$ and $\lim f\left(a_{n}\right)=b_{0}$ exists it is the case that $b_{0} \in E$, then $f$ is holomorphic throughout $D$.

REMARK. In the proof we shall use the same notation and terminology in [1] and [2].

PROOF. Let $S=R-E_{0}$ and let $S_{0}$ be a component of $S$ on which $f$ is nonconstant. It then follows that $S_{0}$ is hyperbolic and that its universal covering surface is $D$. Let $\pi$ denote the projection map of $D$ onto $S_{0}$. Since $\pi$ is a Fatou mapping, the fine limit function $\hat{\pi}$ is defined a.e. on $\partial D$. Here a.e. refers to Lebesgue measure. Since $D$ is a regular covering surface of $S_{0}$, it follows that every point $P^{\prime}$ in $S_{0}$ has a neighborhood $V$ with the property that each component of $\pi^{-1}(V)$ is compact. Hence $\pi$ is of Blaschke type. It follows that for a.e. point

${ }^{1}$ First author is partially supported by NSF Grant GP 8260 .

2 Second author is partially supported by NSF Grant GP 7474. 
$b \in \partial D, \hat{\pi}(b)$ is defined and lies in the minimal Martin boundary of $S_{0}$. Let us now regard $\pi$ as a mapping of $D$ into $R . \pi$ is still a Fatou mapping and hence $\hat{\pi}$ is defined a.e. on $\partial D$. Consequently for a.e. $b \in \partial D$, $\hat{\pi}(b)$ is defined and either lies in $\Delta_{1}$, the minimal Martin boundary of $R$, or else lies in $E_{0}$. Since $f \in H^{P}\left(S_{0}\right), f$ is a Fatou mapping and hence $f \circ \pi$ is a Fatou mapping. Hence $(f \circ \pi)^{\wedge}$ is defined a.e. on $\partial D$. If the set of points $b \in \partial D$ where $\hat{\pi}(b)$ is defined and lies in $E_{0}$, is of positive Lebesgue measure, then the set of points $f(\hat{\pi}(b))=(f \circ \pi)^{\wedge}(b)$ would have to be of positive capacity, since $f$ is nonconstant, by a theorem of Doob; but on the other hand it would have to be of capacity zero since by hypothesis this set would have to be contained in $E$, and $E$ has capacity zero. Hence $\pi$ regarded as a mapping of $D$ into $R$ is of Blaschke type and hence by a theorem of Heins, $E_{0}$ is a set of capacity zero. Hence by a theorem of Parreau, $f$ has a holomorphic extension to $R$.

Remark. Actually, more is true. Thus $R-E_{0}$ is connected and the extended function is of class $H^{P}$ on $R$.

\section{REFERENCES}

1. L. V. Ahlfors and L. Sario, Riemann surfaces, Princeton Univ. Press, Princeton, N. J., 1960.

2. C. Constantinescu and A. Cornea, Ideale Ränder Riemannscher Flächen, Springer-Verlag, Berlin, 1963.

3. J. L. Doob, Conformally invariant cluster value theory, Illinois J. Math. 5 (1961), 521-547.

4. M. Parreau, Sur les moyennes des fonctions harmoniques et analytiques et la classification des surfaces de Riemann, Ann. Inst. Fourier (Grenoble) 3 (1952), 103-197.

5. S. Yamashita, On some families of analytic functions on Riemann surfaces, Nagoya Math. J. 31 (1968), 57-68.

Oregon State University, Corvallis, Oregon 97331 